\title{
BMJ Open Study protocol for a mixed methods exploratory investigation of aftercare services for gestational diabetes in women to develop a new patient-centred model in Germany: the GestDiNa_basic study
}

To cite: Greiner GG, Viehmann A, Linnenkamp U, et al. Study protocol for a mixed methods exploratory investigation of aftercare services for gestational diabetes in women to develop a new patient-centred model in Germany: the GestDiNa basic study. BMJ Open 2021;11:e046048. doi:10.1136/ bmjopen-2020-046048

- Prepublication history and supplemental material for this paper is available online. To view these files, please visit the journal online (http://dx.doi. org/10.1136/bmjopen-2020046048).

Received 20 0ctober 2020 Accepted 06 July 2021

Check for updates

(C) Author(s) (or their employer(s)) 2021. Re-use permitted under CC BY-NC. No commercial re-use. See rights and permissions. Published by BMJ.

For numbered affiliations see end of article.

Correspondence to Gregory Gordon Greiner; Gregory.Greiner@ddz.de

\section{ABSTRACT}

Introduction Women with gestational diabetes mellitus (GDM) have a higher risk of developing type 2 diabetes mellitus compared with women who never had GDM. Consequently, the question of structured aftercare for GDM has emerged. In all probability, many women do not receive care according to the guidelines. In particular, the process and interaction between obstetrical, diabetic, gynaecological, paediatric and general practitioner care lacks clear definitions.

Thus, our first goal is to analyse the current aftercare situation for women with GDM in Germany, for example, the participation rate in aftercare diabetes screening, as well as reasons and attitudes stated by healthcare providers to offer these services and by patients to participate (or not). Second, we want to develop an appropriate, effective and patient-centred care model. Methods and analysis This is a population-based mixed methods study using both quantitative and qualitative research approaches. In various working packages, we evaluate data of the GestDiab register, of the Association of Statutory Health Insurance Physicians of North Rhine and the participating insurance companies (AOK Rheinland/Hamburg, BARMER, DAK Gesundheit, IKK classic, pronova BKK). In addition, quantitative (postal surveys) and qualitative (interviews) surveys will be conducted with randomly selected healthcare providers (diabetologists, gynaecologists, paediatricians and midwives) and affected women, to be subsequently analysed. All results will then be jointly examined and evaluated. Ethics and dissemination The study was approved by the ethics committee of the Faculty of Medicine, Heinrich-Heine-University Düsseldorf (Ethics Committee No.: 2019-738). Participants of the postal surveys and

\section{Strengths and limitations of this study}

- To the best of our knowledge, GestDiNa_basic is the first study providing comprehensive data about aftercare in women with gestational diabetes mellitus (GDM) in Germany.

- Use of a wide spectrum of methods (qualitative and quantitative) and data sources (primary and secondary data), compensating possible reporting or selection bias in each approach, even without data linkage.

- Survey of problem awareness regarding the health consequences of GDM, the practical routines, attitudes and experiences of affected women and healthcare providers potentially involved in the aftercare of women with GDM.

- The study strives for a high representativeness for the healthcare setting in Germany, especially for urban areas, by using nationwide health insurance data. Further, we performed qualitative interviews as well as quantitative surveys among representative samples of patients as well as healthcare providers in a highly populated area in Germany (North Rhine). For some healthcare provider groups all providers in the area will be contacted to participate in the survey. However, primary data derived from a specific area of Western Germany may not be representative for the entirety of Germany or other countries, especially with regard to more rural areas, socioeconomic status, socialisation of the healthcare providers or women's attitudes.

interviews will be informed in detail about the study and the use of data as well as the underlying data protection regulations before voluntarily participating. 


\section{Strengths and limitations of this study}

- A response bias can be expected in the quantitative surveys, for example, if healthcare providers are currently only partially involved in the aftercare (eg, in the group of paediatricians) or if negative feelings such as guilt are involved (eg, in the group of women who did not attend aftercare).

The study results will be disseminated through peer-reviewed journals, conferences and public information.

Trial registration number DRKS00020283.

\section{BACKGROUND}

Around the globe, depending on region, database and definition applied, between $4 \%$ and over $20 \%$ of all women develop hyperglycaemia or gestational diabetes mellitus (GDM) in pregnancy. ${ }^{1-3}$ Women with GDM have a higher risk of developing type 2 diabetes mellitus (T2DM) compared with women who have never had GDM. A recent systematic review along with meta-analysis including 20 studies shows an almost 10 -fold increase in risk of T2DM after pregnancy with GDM. ${ }^{4}$ In addition, affected women have an increased risk of developing the known complications of diabetes, especially cardiovascular diseases. ${ }^{5}$ Moreover, infants born to mothers with GDM are at an increased risk of obesity, with concomitant cardiovascular and/or metabolic disorders. ${ }^{6}$

Given the increased risks associated with GDM, the postpartum period is an important time to monitor women's metabolism. Evidence-based health-related information should be provided, and, in case of impaired glucose regulation, women should be informed about specialised care, for example, additional healthcare providers, diabetes prevention programmes or T2DM disease management programmes (DMPs). ${ }^{78}$

In Germany in 2012, the Federal Joint Committee (G-BA) introduced a GDM screening programme for all pregnant women with the updated maternity directive. Every pregnant woman without a previous diagnosis of diabetes is offered screening by a two-step testing procedure between the 24th and 28th week of gestation. A $50 \mathrm{~g}$ glucose challenge test ('pre-test') should be performed and if values are $\geq 135 \mathrm{mg} / \mathrm{dL}(7.5 \mathrm{mmol} / \mathrm{L})$, a $75 \mathrm{~g}$ oral glucose tolerance test (OGTT, 'diagnostic test') should follow. GDM is diagnosed if blood glucose levels are $\geq 92 \mathrm{mg} / \mathrm{dL}$ ( $5.1 \mathrm{mmol} / \mathrm{L}$ ) before the test (fasting values), $\geq 180 \mathrm{mg} / \mathrm{dL}(10 \mathrm{mmol} / \mathrm{L})$ after 1 hour or $\geq 153 \mathrm{mg} / \mathrm{dL}$ $(8.5 \mathrm{mmol} / \mathrm{L})$ after 2 hours. ${ }^{9}$ Women with diagnosed GDM are recommended to receive an OGTT 6-12 weeks post partum in a specialised medical practice or hospital centre in charge of GDM treatment. The increased risk for women with GDM to develop T2DM requires continuous long-term follow-up with glucose metabolism checks. The National Healthcare Guideline 'Gestational diabetes mellitus (GDM), diagnostics, therapy and aftercare' focuses on screening and diagnosis, treatment, obstetrical care and postpartum care; it recommends checking the glucose metabolism every 2 years by using fasting glucose and glycated haemoglobin or, if necessary, an OGTT. ${ }^{10} 11$ These recommendations are mostly in line with international guidelines. ${ }^{12} 13$ In case of impaired glucose regulation, it is recommended that women receive intensive counselling on lifestyle measures to prevent manifested diabetes; the benefits of lactation are especially emphasised. ${ }^{11}$ However, the German GDM guideline neither mentions any specific programmes nor specifies the disciplines responsible for aftercare, unless overt diabetes is diagnosed. Nevertheless, one study finds that the level of knowledge about the GDM guidelines (from diagnosis to long-term aftercare) and readiness to implement the recommendations in practice among diabetologists and gynaecologists seems satisfactory. ${ }^{14}$

According to international studies, the proportion of women receiving postpartum blood glucose monitoring is too low. ${ }^{15-17}$ In the densely populated North Rhine region (Germany), approximately $40 \%$ of women with GDM who receive care in specialised diabetes practices, which take part in the German GestDiab register (https://www.windiab.de/gestdiab/), show up for GDM aftercare at the practice. ${ }^{15}$ The percentage of participation in the diabetes screening 6-12 weeks post partum differs widely $(6 \%-100 \%$, median $48 \%)$ between the practices, presumably due to different scheduling strategies, for example, recall systems or early appointment allocation. ${ }^{18} \mathrm{~A}$ multicentre study from three different German clinics with diabetes and pregnancy specialist services shows $51 \%$ participation in GDM aftercare overall. However, the authors report large differences between the centres, which might be due to the composition of the population as well as to differences in the care environment. ${ }^{19}$

Though we do not know the proportion of women in Germany receiving diabetes screening 6-12 weeks post partum from other healthcare providers than the diagnosing one, we assume that participation rate in postpartum glucose testing is lower throughout the country compared with the rates of the GestDiab register. In Germany, little is known about the factors influencing the participation rate in GDM aftercare, neither regarding women concerned nor healthcare professionals involved. International studies show that higher socioeconomic status of women with GDM (better education, higher income) is associated with higher aftercare attendance; geographical location, age, ethnicity and quality of care during pregnancy also play a role. ${ }^{20-22}$ In Germany, it is unknown if women who do not attend GDM aftercare are merely unaware of it or consciously opt out. To develop a structured GDM aftercare programme, more information is needed concerning knowledge and opinions of the women affected regarding the importance of GDM aftercare in the postpartum period and later. In addition, information on experiences, attitudes and beliefs regarding responsibilities need to be surveyed and analysed among healthcare providers. A wide network 


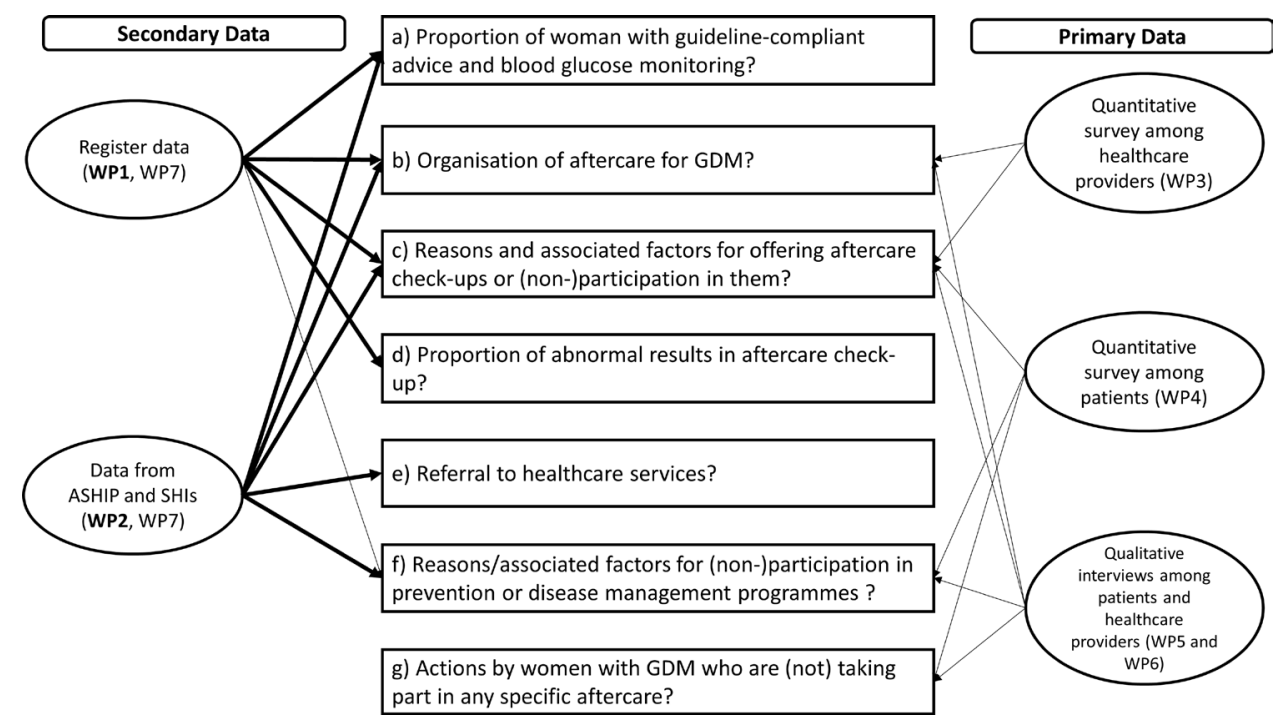

Figure 1 Overview of data sources, research questions and working packages in GestDiNa_basic. ASHIP, Association of Statutory Health Insurance Physicians of North Rhine; GDM, gestational diabetes mellitus; SHI, statutory health insurance; WP, working package.

of professionals must be considered: professionals at maternity wards, midwives, diabetologists, general practitioners, gynaecologists and paediatricians.

\section{Objectives}

The aim of our study is to analyse the current aftercare situation of women with GDM in Germany. We will use data from the GestDiab register (established in 2008, it is the largest register in Germany that systematically collects data on diabetes and pregnancy ${ }^{15}$ ), as well as data from five statutory health insurances (AOK Rheinland/Hamburg, BARMER, DAK Gesundheit, IKK classic and pronova BKK), and from the Association of Statutory Health Insurance Physicians (ASHIP) of North Rhine. This allows us to assess the participation in GDM aftercare and factors affecting it. Furthermore, qualitative interviews as well as quantitative surveys will help to analyse problem awareness as well as concepts, attitudes, routines and experiences of healthcare providers regarding GDM aftercare. Similarly, quantitative surveys and qualitative interviews will be used to assess problem awareness, knowledge and experiences of women with GDM. Based on the results, we will develop a care model.

The key research questions of the GestDiNa_basic project are (figure 1):

(a) How many women receive guideline-compliant advice and blood glucose monitoring after a pregnancy accompanied by GDM?

(b) How is the aftercare of women with GDM organised? This concerns the typical pathways (utilisation; time and place of blood glucose monitoring and/or counselling; actors and their coordination/communication; referrals; documentation; assumption of responsibility; continuity and duration), including all actors in the field of GDM.

(c) What are the reasons, attitudes and associated factors influencing whether healthcare providers offer aftercare diabetes screening and whether women with GDM participate?

Moreover, we wish to exploratively investigate:

(d) What is the proportion of impaired glucose tolerance, impaired fasting glucose or T2DM found by the postpartum OGTT (ppOGTT), and what factors are associated with impaired glucose regulation?

(e) How often are women with impaired glucose regulation advised to use care services (prevention programmes, DMPs)?

(f) What reasons do women with diagnosed GDM provide for (non)participating in prevention or DMPs, and what are the associated factors?

(g) What measures are taken by women with diagnosed GDM after delivery with and without specific aftercare glucose testing (eg, breast feeding, diet, exercise)?

\section{METHODS}

\section{Patient and public involvement}

A patient representative as well as several healthcare providers are part of the study consortium, functioning as joint grant holders or co-applicants of the study. Jointly with co-researchers (women with former GDM and citizens), they have been involved in the development of the study design and will be involved in carrying out the study, as well as in interpreting, publishing and disseminating the results.

\section{General design}

GestDiNa_basic (short for Nachsorge bei Gestationsdiabetes: aftercare for gestational diabetes) is a multicentre, noninterventional study following a mixed methods approach with a duration of 3years lasting from September 2019 until August 2022. Data collection, recruitment of participants and analyses are grouped into eight working packages (WPs). 
We adhere to best practice guidelines for STandardized Reporting Of Secondary data Analyses, Consolidated Health Economic Evaluation Reporting Standards, Strengthening the Reporting of Observational Studies in Epidemiology and Standards for Reporting Qualitative Research.

\section{Data sources, participants and sampling}

This study uses mixed methods and a wide range of data sources for answering the complex study questions. Data from the different sources will not be linked for participants; instead, findings will be derived by all actors of the study group scientifically examining the data.

\section{Working packages}

WP1, based on registry data: In WP1, we are addressing research questions (a), (b), (c) and (d) (figure 1), using data of the GestDiab register hosted by the winDiab $\mathrm{gGmbH}$ (Wissenschaftliches Institut der niedergelassenen Diabetologen: Scientific institute of resident diabetologists). Participating specialised diabetes practices (DSPs) provide data of women with diagnosed GDM via an online tool. Information includes, for example, their medical history, date and mode of childbirth, metabolic parameters, lifestyle factors and medication. A full list of available and considered variables can be found in online supplemental appendix A. In 2016, 58 practices participated, providing information on 4921 women. The practices are located all over Germany, with a focus in the North Rhine area. All women gave informed consent enabling the DSPs to share their data.

We will use the data available from 2015 to 2020. Based on previous experiences from working with the register, we estimate a sample size of about 25000-30 000 patients with diagnosed GDM.

WP2, based on data from the five statutory health insurances and the ASHIP:

We are addressing research questions (a), (b), (c), (e) and (f) (figure 1), using data of five statutory health insurances (SHI) in Germany, with a total number of approximately 21 million insured people, as well as data of the ASHIP of North Rhine (KV Nordrhein). These data are fully pseudonymised and contain medical diagnoses, outpatient and inpatient care, as well as received medication covering the years 2014-2020.

Women with at least one documented GDM diagnosis during the observation period are included in the analysis. Excluded from the analysis are women with manifested diabetes before pregnancy, women who switched to another insurance within 1 year before the quarter in which the pregnancy started or women with a pregnancy lasting less than 26 weeks.

WP3, based on a quantitative survey among healthcare providers:

We are conducting a postal survey among healthcare providers to answer parts of research question (b) and (c) (figure 1). Healthcare providers in the region of
North Rhine are randomly selected from the ASHIP by age and gender in order to obtain a representative sample for Germany. Midwives, for whom the ASHIP has no address list, will be contacted by cooperation partners. Gynaecologists working in hospitals are not registered in the ASHIP, either. A list of hospitals in the North Rhine region will be compiled and 50 clinics will be contacted, with all administrative districts and the different equipment of the clinics (the presence of a neonatal ward, a perinatal centre, etc) represented as equally as possible. In total, we aim to contact 2629 healthcare providers of different professional groups: 1000 general practitioners, 500 gynaecologists, 766 paediatricians (complete survey), 163 diabetologists (complete survey), 100 midwives and 100 gynaecologists working in hospitals.

WP4, based on a quantitative survey among women with GDM who did not attend aftercare:

This WP contributes to answering our research questions (c), (f) and (g) (figure 1). We are conducting a postal questionnaire survey among women with GDM who did not attend aftercare ppOGTT. In total, the GestDiab register counts around 3000 women per year not taking part in aftercare ppOGTT at the participating DSP where the pregnancy was supervised. Around 10\% $(n=300)$ of the patients from the GestDiab register who did not attend ppOGTT within a year will be randomly selected and asked to participate in the study.

$W P 5+W P 6$, based on qualitative interviews with healthcare providers (WP5) and women with GDM (WP6):

Our aim in this WP is to answer parts of research questions (b), (c), (f) and (g) (figure 1). Trained researchers are conducting open and semi-structured face-to-face interviews (or small focus-group interviews) with healthcare providers of different specialisations and women with GDM. Healthcare providers will be recruited through professional networks. From experience, we assume that saturation will be reached after about 50 interviews (WP5). Women with GDM will be recruited through gynaecologists, maternity clinics and DSPs. From experience, we assume that saturation will be reached after about 25 interviews (WP6).

$W P 7$, based on longitudinal analyses of register and secondary data:

To tackle research questions (d), (e) and (f) (figure 1), we will follow-up women with diagnosed GDM up to 2 years or a following pregnancy after delivery, using the same data basis and items as in WP1 (registry data) and WP2 (SHI data), but supplemented by more recent years. We aim at analysing the proportion of women with impaired glucose regulation and the proportion of affected women that are referred to prevention programmes or DSPs. Furthermore, we will investigate factors associated with impaired glucose regulation and the use of specific prevention programmes and DSPs.

WP8, based on the combination of the results:

In WP8, we will combine the insights of the WPs $1-7$ to develop an optimised care model for GDM aftercare in Germany. 
The aim of WP8 is to identify facilitating and hindering factors for guideline-compliant aftercare from the analysis of the current care situation (WPs 1 and 2) and the experiences and attitudes of stakeholders and women (WPs 3-6) to develop a complex, patient-centred care model. For this purpose, the study results are evaluated by the study team in multidisciplinary groups with experts from the care sector and patient representatives by means of a force field analyses. Concrete measures for optimising care will be developed in a consensus process. In addition to measures to optimise screening and monitoring, the focus will also be on the development of measures to enhance women's health literacy.

\section{Data analysis and instruments}

In WP1, we are analysing the proportion of women who received a ppOGTT as well as associated factors such as age, sociodemographic factors and prescribed medication. A full list can be found in online supplemental appendix A. We also analyse associated factors for practices such as number of patients per year or region. The proportions will be cluster-adjusted with 95\% CIs; the calculation will use univariate and multiple regression models. Methods from survival analysis (Kaplan-Meier estimates, Cox regression models) will be used to assess time to the respective events.

Statistical analyses of WP2 are similar to WP1. Diagnoses and accounting codes (Einheitlicher Bewertungsmaßstab (EBM)) will define relevant outcomes. The uniform evaluation standard EBM is the remuneration system of contract medical and psychotherapeutic care in Germany, which determines the content of billable services. To identify women with delivery, the relevant German diagnosis-related groups are $\mathrm{O} 01 \mathrm{~A}$ to $\mathrm{O} 01 \mathrm{H}$ for different types of caesarean section, and O02A to O02B, as well as O60A to O60D, for vaginal delivery. Furthermore, OPS-Codes (Operation and Procedure Classification System, the German modification of the International Classification of Procedures in Medicine) 5-72 to 5-75 will be used. Length of pregnancy will be identified via the International Statistical Classification of Diseases and Related Health Problems (ICD) ICD-10-code O09. To identify women with diabetes mellitus type 1 or type 2 , we will use the ICD-10-codes E10 to E14 and O24. Gestational diabetes will be identified via ICD-10-code O24.4. We consider, for example, age, education status, prescription of insulin during pregnancy, obesity, non-alcoholic fatty liver disease, hyperlipidaemia and hypertension as potential moderating factors. We are not able to consider, for example, the prior number of pregnancies or diagnoses because we do not have information for women before 2014 (start of the observation period).

In WP3, we use descriptive statistics to analyse the quantitative survey of healthcare providers. They will be asked about their awareness of health consequences of GDM, their practical routines as well as attitudes towards postpartum aftercare. Each group of healthcare providers will receive a different version of the questionnaire including a joint section. Thus, we consider their different roles in the field of GDM aftercare while also enabling comparisons between the groups. The questionnaires are inspired by a study by Groten $e t a l^{14}$ and adapted to the research questions in a multiprofessional team. Furthermore, they were comprehensively pretested in cognitive interviews with representatives of different relevant healthcare professions. The validated final German questionnaires for each healthcare profession are attached in online supplemental appendix B and will be discussed in detail in future publications.

For WP4, the quantitative survey of women with GDM, we mainly perform descriptive statistics. The survey contains questions on the sociodemographic background, reasons for not attending aftercare, specific postpartum behaviours like lifestyle changes, breast feeding and participation in prevention programmes or DMPs in general. It also asks whether follow-up examinations were carried out by other healthcare providers. For the development of the questionnaire, we use and adapt established questionnaires and item batteries-for example, the German Health Survey, the DEGS study (German Health Interview and Examination Survey for Adults) and the KiGGS study (German Health Interview and Examination Survey for Children and Adolescents) of the Robert Koch Institute. ${ }^{23-25}$ Similar to WP3, the questionnaire will be extensively pretested in cognitive interviews with women who had had GDM. Future publications will report in detail about the questionnaire for women with GDM.

In WP5 and WP6, in qualitative guided interviews with healthcare providers and women with GDM, we ask about principles, attitudes, self-reported routines and experiences with GDM aftercare. Furthermore, we want to explore the needs and requirements of affected women as well as the resources, incentives and barriers for both participation (patients' perspective) and supply (healthcare providers' perspective). In addition, we wish to explore if women feel well-informed about their GDM, its risks and potential care provision. Furthermore, we will learn about their information needs, sources and selfcare strategies. For the qualitative interviews of healthcare providers and women with GDM, content analyses of verbatim transcripts will be performed using open, inductive coding in a multidisciplinary team consisting of scientists with different fields of expertise, women with GDM experience and healthcare providers. In a first step, codes and categories will be developed from the interviews in each group individually. In a second step, findings will be made comparable between the different professional groups interviewed. The results will be revalidated with professionals from each group. Future publications will discuss in detail about the guidelines for the interviews.

In WP7, we will estimate the cumulative incidences for the occurrence of postpartum hyperglycaemia and calculate time-dependent event probabilities with survival methods such as the Kaplan-Meier plot for the time of diagnosis. We will perform Cox regression 
models to analyse possible associated factors. In addition, we shall use descriptive statistics to analyse the utilisation of different aftercare services, for instance, prevention programmes such as weight reduction regimens or DMPs. We also aim to analyse which and how many healthcare providers are involved in aftercare up to 2 years after childbirth.

In WP8, we aim at developing an overarching concept for an adequate, effective and patient-centred care model that would be applicable nationwide. In the first step, the results of the specific subgroups shall be summed up and discussed. The identified barriers and resources for aftercare will be visualised and weighted in group-specific force field analyses. In addition, the results are to be compared with the current aftercare landscape. Representatives of all study populations will be incorporated as experts in these integration analyses. This enables the development of a tailored supply and aftercare model, which is essential for effective short-term and long-term aftercare. In a second step, all study members will integrate the insights of step one as well as the literature in one system model. This system model shall relate the weighted barriers and resources for each subgroup to the current aftercare system.

\section{ETHICS}

The study GestDiNa_basic was approved by the ethics committee of the Heinrich Heine University Düsseldorf (Ethics Committee No.: 2019-738) and is registered in the German Registry for Clinical Trials (Deutsches Register Klinischer Studien). The GestDiab register was approved by the ethics committee of the Medical Association of North Rhine (Ethics Committee No.: 2019272). The use of register data as well as secondary and primary data are in line with the common data protection regulations. A central issue is the need to encode or anonymise data. Participants of the postal surveys and interviews will be informed in detail about the study, the use of data and the underlying data protection regulations before voluntary filling out the questionnaires or attending the interviews. No personally identifiable information will be saved during data collection to maintain the anonymity of the study participants. Any information allowing for personal identification that was collected unintentionally will be removed from the data immediately. The processing and use of register data, insurance-related data and primary data should guarantee integrity (eg, protection against intentional or negligent misrepresentation of programmes or manipulation of data), confidentiality (eg, protection against unauthorised access) and availability (eg, protection against theft or destruction).

\section{DISSEMINATION}

With the dissemination of the instruments and the results in this and future publications, we want to reach the scientific community as well as a diverse range of relevant policymakers, stakeholder groups, patients and the interested public at the local, national and international level. Therefore, results from the study will be disseminated in peer-reviewed journals and at conferences. In addition, press releases, social media and other dissemination channels are integrated into the communication concept. The authorship of any papers related to this study will follow the ICMJE (International Committee of Medical Journal Editors) recommendations. ${ }^{26}$

\section{DISCUSSION}

To the best of our knowledge, this is the first study that will provide comprehensive data about aftercare in women with GDM in Germany. These data will enable betterinformed policy decisions on health and care. Additionally, it includes representative samples of all healthcare providers potentially involved in the aftercare of women with GDM, which allows the integration of the planned healthcare model. We assume that the topic of GDM aftercare is currently being addressed particularly by gynaecologists and diabetologists in charge and, possibly, by general practitioners. However, maternity wards, outpatient midwives involved in postpartum care and paediatricians are also potentially important and well-suited for GDM aftercare-and they receive very little attention so far. Alternatively, patients with post-GDM could be contacted through the SHIs, who may have all the relevant data identifying the target group. GDM and its aftercare are therefore considered from different perspectives, which are to be brought together in the consortium and used for planning future care.

It is a strength of this study that it covers a wide spectrum of methods, data sources and participants: qualitative and quantitative methods are used; all groups of healthcare providers in the field of GDM are investigated; different data sources are used enabling important analyses on patients' and healthcare providers' side. In addition to data on the current situation of GDM aftercare services, the study will provide reliable information on the prevalence of GDM in Germany, which is lacking so far.

\section{Author affiliations}

${ }^{1}$ Institute for Health Services Research and Health Economics, Centre for Health and Society (chs), Medical Faculty and University Hospital Düsseldorf, Heinrich Heine University Düsseldorf, Düsseldorf, Nordrhein-Westfalen, Germany ${ }^{2}$ Institute for Health Services Research and Health Economics, German Diabetes Center, Leibniz Institute for Diabetes Research at Heinrich Heine University Düsseldorf, Düsseldorf, Nordrhein-Westfalen, Germany

${ }^{3}$ Department of Gynecology and Obstetrics, University Hospital, Heinrich Heine University Düsseldorf, Düsseldorf, Nordrhein-Westfalen, Germany ${ }^{4}$ Institute of General Practice, Centre for Health and Society (chs), Medical Faculty, Heinrich Heine University Düsseldorf, Düsseldorf, Nordrhein-Westfalen, Germany ${ }^{5}$ Institute for Biometrics and Epidemiology, German Diabetes Center Leibniz Institute for Diabetes Research at Heinrich Heine University Düsseldorf, Düsseldorf, Nordrhein-Westfalen, Germany

${ }^{6}$ Institute of Child Nutrition, Max Rubner-Institute Federal Research Institute of Nutrition and Food, Karlsruhe, Baden-Württemberg, Germany 
${ }^{7}$ Department of General Paediatrics, Neonatology, and Paediatric Cardiology, University Children's Hospital, Medical Faculty, Heinrich Heine University Düsseldorf, Düsseldorf, Nordrhein-Westfalen, Germany

${ }^{8}$ Department of Applied Heath Sciences, Bochum University of Applied Sciences, Bochum, Nordrhein-Westfalen, Germany

${ }^{9}$ PMV Research Group, Faculty of Medicine and University Hospital Cologne, University of Cologne, Cologne, Nordrhein-Westfalen, Germany

${ }^{10}$ winDiab gGmbH, Düsseldorf, Nordrhein-Westfalen, Germany

${ }^{11}$ Association of Statutory Health Insurance Physicians of North Rhine (KV Nordrhein), Düsseldorf, Nordrhein-Westfalen, Germany

${ }^{12}$ BARMER, Statutory Health Insurance, Wuppertal, Nordrhein-Westfalen, Germany

${ }^{13}$ DAK Gesundheit, Statutory Health Insurance, Hamburg, Germany

${ }^{14}$ pronova BKK, Statutory Health Insurance, Ludwigshafen, Rheinland-Pfalz, Germany

${ }^{15}$ AOK Rheinland/Hamburg, Statutory Health Insurance, Düsseldorf, NordrheinWestfalen, Germany

${ }^{16}$ IKK classic, Statutory Health Insurance, Münster, Nordrhein-Westfalen, Germany

${ }^{17}$ Patient Representative, Dortmund, Nordrhein-Westfalen, Germany

${ }^{18}$ German Center for Diabetes Research, Neuherberg, Bayern, Germany

\section{Acknowledgements We thank all participants for the support of our study.}

Collaborators Verena Leucht (Institute of General Practice, Centre for Health and Society (chs), Medical Faculty, Heinrich Heine University Düsseldorf, Düsseldorf, Germany), Jacqueline Verena Warth (Institute of General Practice, Centre for Health and Society (chs), Medical Faculty, Heinrich Heine University Düsseldorf, Düsseldorf Germany), Franziska Meier-Stiegen (Department of Gynecology and Obstetrics, University Hospital, Heinrich Heine University Düsseldorf, Düsseldorf, Germany), Gabriele Bizjak (Department of Gynecology and Obstetrics, University Hospital, Heinrich Heine University Düsseldorf, Düsseldorf, Germany), Felix Borgmeier (Department of Gynecology and Obstetrics, University Hospital, Heinrich Heine University Düsseldorf, Düsseldorf, Germany), Soner Öner-Sieben (Institute of Child Nutrition, Max Rubner-Institute Federal Research Institute of Nutrition and Food, Karlsruhe, Baden-Württemberg, Germany, Department of General Paediatrics, Neonatology and Paediatric Cardiology, University Children's Hospital, Medical Faculty, Heinrich Heine University Düsseldorf, Düsseldorf, Germany), Dietmar Weber (winDiab gGmbH, Düsseldorf, Germany), Manuela Behling (winDiab gGmbH, Düsseldorf, Germany), Martina Gierse (winDiab gGmbH, Düsseldorf, Germany), Kerstin Merker (winDiab gGmbH, Düsseldorf, Germany), Judith Klein (winDiab $\mathrm{gGmbH}$, Düsseldorf, Germany), Christian Graf (BARMER, Statutory Health Insurance, Wuppertal, Germany), Thomas Abele (BARMER, Statutory Health Insurance, Wuppertal, Germany), Martin Schneider (BARMER, Statutory Health Insurance, Wuppertal, Germany), Joachim Saam (BARMER, Statutory Health Insurance, Wuppertal, Germany), Stefanie Wobbe-Robinski (DAK Gesundheit, Statutory Health Insurance, Hamburg, Germany), Olga Dortmann (AOK Rheinland/Hamburg, Statutory Health Insurance, Düsseldorf, Germany), Tim Hollmann (IKK classic, Statutory Health Insurance, Münster, Germany).

Contributors GGG, AV, ULi and VLe drafted the manuscript. Al is the chief investigator and together with SW, OK, TF, RE, ULa, PI, MK, MT, BW, SS, IS-L, CJR, FM and UJ initiated the project and led the grant proposal. GGG, AV, ULi, VLe, MN, LS, DM-B, VLa, HA, VG, NW-H, TK, SC, BK and SK-S made substantial contributions to the conception and design of the project. MN, OK, VLa and PI are in charge of the statistical analysis. HA and MK are in charge for acquisition and management of registry data. PI, VLa, MT, VG, BW, NW-H, SS, TK, IS-L, SC, CJR, BK, FM and SK-S are in charge for acquisition, application for use and management of SHI data. GGG AV, ULi, SW, VLe, TF, RE, LS, ULa, DM-B, HA and MK are in charge for acquisition of primary data. GGG, SW, VLe, PI and Al are supervising and leading the consortium. All authors and collaborators participated in the preparation of the study and are part of the multiprofessional team and the GestDiNa study group. All authors and collaborators critically revised the manuscript's drafts and approved the final version of the manuscript.

Funding This work is supported by the Innovation Fund coordinated by the Innovation Committee of the Federal Joint Committee (G-BA) in Germany; grant number: 01VSF18009.

Disclaimer The funder has no role in study design, data collection and analysis, decision to publish, or preparation of any kind of manuscript.

Competing interests None declared.

Patient consent for publication Not required.

Provenance and peer review Not commissioned; externally peer reviewed.
Supplemental material This content has been supplied by the author(s). It has not been vetted by BMJ Publishing Group Limited (BMJ) and may not have been peer-reviewed. Any opinions or recommendations discussed are solely those of the author(s) and are not endorsed by BMJ. BMJ disclaims all liability and responsibility arising from any reliance placed on the content. Where the content includes any translated material, BMJ does not warrant the accuracy and reliability of the translations (including but not limited to local regulations, clinical guidelines, terminology, drug names and drug dosages), and is not responsible for any error and/or omissions arising from translation and adaptation or otherwise.

Open access This is an open access article distributed in accordance with the Creative Commons Attribution Non Commercial (CC BY-NC 4.0) license, which permits others to distribute, remix, adapt, build upon this work non-commercially, and license their derivative works on different terms, provided the original work is properly cited, appropriate credit is given, any changes made indicated, and the use is non-commercial. See: http://creativecommons.org/licenses/by-nc/4.0/.

ORCID iD

Gregory Gordon Greiner http://orcid.org/0000-0001-7341-0818

\section{REFERENCES}

1 IQTIG - Institut für Qualitätssicherung und Transparenz im Gesundheitswesen. Bundesauswertung zum Erfassungsjahr 2017 Geburtshilfe Qualitätsindikatoren, 2018.

2 Guariguata L, Linnenkamp U, Beagley J, et al. Global estimates of the prevalence of hyperglycaemia in pregnancy. Diabetes Res Clin Pract 2014;103:176-85.

3 Melchior H, Kurch-Bek D, Mund M. The prevalence of gestational diabetes. Dtsch Arztebl Int 2017;114:412-8.

4 Vounzoulaki E, Khunti K, Abner SC, et al. Progression to type 2 diabetes in women with a known history of gestational diabetes: systematic review and meta-analysis. BMJ 2020;369:m1361.

5 Shostrom DCV, Sun Y, Oleson JJ, et al. History of gestational diabetes mellitus in relation to cardiovascular disease and cardiovascular risk factors in US women. Front Endocrinol 2017;8:144

6 Zhu Y, Olsen SF, Mendola P, et al. Growth and obesity through the first $7 \mathrm{Y}$ of life in association with levels of maternal glycemia during pregnancy: a prospective cohort study. Am J Clin Nutr 2016;103:794-800.

7 Middleton P, Crowther CA. Reminder systems for women with previous gestational diabetes mellitus to increase uptake of testing for type 2 diabetes or impaired glucose tolerance. Cochrane Database Syst Rev 2014;3:CD009578.

8 Tieu J, McPhee AJ, Crowther CA, et al. Screening and subsequent management for gestational diabetes for improving maternal and infant health. Cochrane Database Syst Rev 2014;2:CD007222.

9 Metzger BE, Gabbe SG, Persson B, et al. International association of diabetes and pregnancy study groups recommendations on the diagnosis and classification of hyperglycemia in pregnancy: response to Weinert. Diabetes Care 2010;33:e98-82.

10 Kleinwechter H, Schäfer-Graf U, Bührer C. Gestational diabetes mellitus (GDM) evidence based guideline on diagnosis, management, and aftercare of the German diabetes association (DDG) and the German association of gynecology and obstetrics (DGGG). Diabetologie und Stoffwechsel 2011;6:290-328.

11 Schäfer-Graf U, Laubner K, Hummel S, et al. Gestationsdiabetes mellitus (GDM), Diagnostik, therapie und Nachsorge. Diabetologie und Stoffwechsel 2018;13:S174-84

12 American Diabetes Association. 14. Management of Diabetes in Pregnancy: Standards of Medical Care in Diabetes-2020. Diabetes Care 2020;43:S183-92.

13 Zhang M, Zhou Y, Zhong J, et al. Current guidelines on the management of gestational diabetes mellitus: a content analysis and appraisal. BMC Pregnancy Childbirth 2019;19:200.

14 Groten T, Schmitz S, Schippert C, et al. Knowledge and implementation of the S3 guideline on gestational diabetes among Gynecologists and Diabetologists four years after publication. Geburtshilfe Frauenheilkd 2016;76:771-8.

15 Adamczewski H, Weber D, Heinemann L. Betreuung von schwangeren Frauen in diabetologischen Schwerpunktpraxen. Diabetes Stoffw Herz 2010;19:99-109.

16 Blatt AJ, Nakamoto JM, Kaufman HW. Gaps in diabetes screening during pregnancy and postpartum. Obstet Gynecol 2011;117:61-8.

17 Zera CA, Bates DW, Stuebe AM, et al. Diabetes screening reminder for women with prior gestational diabetes: a randomized controlled trial. Obstet Gynecol 2015;126:109-14. 
18 Adamczewski H, Weber D, Faber-Heinemann G. Einfluss Der Gestationsdiabetes-Leitlinie Der DDG auf die Versorgungsrealität: Analysen des register GestDiab. Diabetologie und Stoffwechsel 2016;11:341-9.

19 Schaefer-Graf UM, Klavehn S, Hartmann R, et al. How do we reduce the number of cases of missed postpartum diabetes in women with recent gestational diabetes mellitus? Diabetes Care 2009;32:1960-4.

20 Eggleston EM, LeCates RF, Zhang F, et al. Variation in postpartum glycemic screening in women with a history of gestational diabetes mellitus. Obstet Gynecol 2016;128:159-67.

21 Nielsen KK, Kapur A, Damm P, et al. From screening to postpartum follow-up - the determinants and barriers for gestational diabetes mellitus (GDM) services, a systematic review. BMC Pregnancy Childbirth 2014;14:41.
22 Tovar A, Chasan-Taber L, Eggleston E, et al. Postpartum screening for diabetes among women with a history of gestational diabetes mellitus. Prev Chronic Dis 2011;8:A124.

23 Beitz R, Bergmann E, Bergmann KE, et al. BundesGesundheitssurvey: Bausteine Der Gesundheitssurveillance in Deutschland, 2003.

24 Gößwald A, Lange M, Kamtsiuris P, et al. Degs: studie Zur Gesundheit Erwachsener in Deutschland. Bundesgesundheitsblatt Gesundheitsforschung Gesundheitsschutz 2012;55:775-80.

25 Hölling H, Schlack R, Kamtsiuris P. BundesgesundheitsblattGesundheitsforschung-Gesundheitsschutz 2012;55:836-42.

26 International Committee of Medical Journal Editors. Defining the role of authors and contributors, 2017. 